\title{
A integração ensino-serviço na formação de enfermeiros e médicos: a experiência da FAMEMA
}

\author{
School-service integration in the training of nurses and doctors: \\ the experience of FAMEMA
}

Maria José Sanches Marin ${ }^{1}$

Maria Amélia de Campos Oliveira ${ }^{2}$

Márcia Aparecida Padovan Otani ${ }^{1}$

Cristina Peres Cardoso ${ }^{1}$

Maria Yvette Aguiar Dutra Moravcik ${ }^{3}$

Lucieni de Oliveira Conterno ${ }^{3}$

Luzmarina Aparecida Doretto Braccialli ${ }^{1}$

Cássia Regina Rodrigues Nunes ${ }^{1}$

Antonio Carlos Siqueira Júnior ${ }^{1}$

${ }^{1}$ Curso de Enfermagem, Faculdade de Medicina de Marília. R. Monte Carmelo 800, Fragata. 17.519-030 Marilia SP Brasil. marnadia@terra.com.br ${ }^{2}$ Escola de Enfermagem, Universidade de São Paulo. ${ }^{3}$ Curso de Medicina da Faculdade de Medicina de Marília.
Abstract The scope of this study is to identify contributions and limits of teaching-service integration between the School of Medicine of Marilia and the Municipal Health Department of Marilia for academia, for the health teams and for the community. The study involved cross-sectional research using a quantitative approach. A questionnaire with 20 affirmations structured around a Likert scale was applied to students, professors and collaborating professors with a total of 183 participants. A trend for a positive evaluation of teaching-service integration for professional education was revealed. As regards contributions of teaching-service integration to the community, the positive evaluation was close to $50 \%$. The items referring to contributions to the health teams were the ones that had the highest number of negative responses. Data indicate that it is necessary to move forward such that teaching and service are considered interdependent processes with possibilities of joint reflections.

Key words Teaching-care integration services. Education, Health services for students, Human resource training
Resumo O estudo tem como objetivo identificar contribuições e limites da integração ensino-serviço entre a Faculdade de Medicina de Marília e a Secretaria Municipal de Saúde de Marília, para a academia, para as equipes de saúde e para a comunidade. Trata-se de uma pesquisa transversal, de abordagem quantitativa. Um questionário com 20 assertivas, organizado sob a forma de escala Likert, foi aplicado a estudantes, docentes e professores colaboradores, num total de 183 participantes. Constatou-se tendência à avaliação positiva da integração ensino-serviço para a formação profissional. Quanto às contribuições da integração ensino-serviço para a comunidade, a avaliação positiva aproximou-se de 50\%. Os itens referentes às contribuições para as equipes de saúde foram os que tiveram maior quantidade de respostas negativas. Os dados indicam que é preciso avançar de forma que ensino e serviço sejam considerados processos interdependentes e com possibilidades de reflexões conjuntas.

Palavas-chave Serviços de integração docente assistencial, Ensino, Serviços de saúde para estudantes, Formação de recursos humanos 


\section{Introdução}

A proposta de articulação entre o ensino e os serviços de saúde no processo de formação profissional é antiga, tendo sido motivo de experimentações variadas, algumas das quais resultaram em avanços ao longo das últimas décadas no Brasil. Atualmente, essa articulação vem sendo enfatizada pelo Ministério da Saúde como uma estratégia importante para a formação de profissionais que atendam aos princípios e diretrizes do Sistema Único de Saúde (SUS) ${ }^{1,2}$.

A Constituição brasileira de 1988 confere ao SUS a responsabilidade de regular a formação dos profissionais da saúde. O inciso III do artigo 200 afirma textualmente que ao SUS compete ordenar a formação de recursos humanos na área de saúde ${ }^{3}$. A consolidação do SUS requer a implementação de propostas e de estratégias que viabilizem a política de formação da força de trabalho em saúde ${ }^{4}$.

Em vista disso, o Ministério da Saúde vem incentivando propostas que visam à inovação dos processos de ensino e aprendizagem e à articulação destes com os serviços de saúde para intervenção no processo saúde-doença sob a lógica da vigilância à saúde. Por meio delas, busca-se alcançar a formação de profissionais críticos, cidadãos, preparados para aprender, criar, propor e construir um novo modelo de atenção à saúde ${ }^{5}$.

Para tanto, o processo de formação deve ocorrer de forma articulada com o mundo do trabalho, com ênfase no desenvolvimento de profissionais crítico-reflexivos, com vistas à transformação das práticas em saúde ${ }^{6}$. Como as práticas profissionais devem ser organizadas para responder às necessidades de saúde da população, a aproximação entre a academia e os serviços de saúde há de levar em conta o reconhecimento de tais necessidades no cotidiano do trabalho em saúde na Atenção Básica para efetivar os princípios e as diretrizes do SUS7.

A Faculdade de Medicina de Marília (Fame$\mathrm{ma})$, há mais de uma década, vem caminhando nessa direção, por meio do desenvolvimento curricular de seus cursos de graduação em Enfermagem e em Medicina, que se caracterizam pela integração tanto entre as disciplinas, como entre ensino-serviço e teoria-prática. Essa construção curricular integrada é desenvolvida com a utilização de metodologias ativas de aprendizagem.

Nos projetos político-pedagógicos da Famema, optou-se pela definição de currículo como um projeto seletivo de cultura, cultural, social, politica e administrativamente condicionado, que preenche a atividade escolar e que se torna realidade dentro das condições da escola tal como se acha configurada ${ }^{8}$. Para ser emancipatório, o currículo necessita ser entendido como práxis e, portanto, como um mediador entre a sociedade exterior às escolas e as práticas sociais concretas que nelas se exercitam como consequência do desenvolvimento do currículo ${ }^{8}$.

Concomitantemente às mudanças ocorridas no ensino da Famema, houve reestruturação dos serviços da Atenção Básica à Saúde do município de Marília, com a implantação de 31 equipes de Saúde da Família, para atuar na lógica da Vigilância à Saúde. Com isso, as unidades da Estratégia Saúde da Família (ESF) passaram a ser um espaço privilegiado para o ensino e também para a transformação da prática profissional.

$\mathrm{Na}$ busca de favorecer a visão ampliada de saúde e a formação de sujeitos ativos, comprometidos com a transformação da realidade, desde a década de 80, a Famema e a Secretaria Municipal de Saúde de Marília (SMSM) vêm trabalhando em parceria por meio do projeto de integração docente-assistencial, fortalecido posteriormente com o projeto UNI-Marília.

Na sequência, o projeto Famema Século XXI, vinculado ao Promed, propiciou a inserção de docentes e de estudantes nos serviços locais de saúde, passando a desenvolver em unidades da ESF do Município a maior parte das atividades educacionais da $1^{\mathrm{a}}, 2^{\mathrm{a}}$ e $4^{\mathrm{a}}$ séries dos cursos de Medicina e Enfermagem. Mais recentemente, esse movimento vem sendo subsidiado por projetos vinculados ao Ministério da Saúde, quais sejam, o Pró-Saúde e o PET-Saúde, que favorecem a integração ensino-serviço com ênfase em ações educativas voltadas para a promoção da saúde.

Para o desenvolvimento dos currículos atuais dos Cursos de Enfermagem e Medicina da Famema, os profissionais do serviço (médicos e enfermeiros) são inseridos na construção, no desenvolvimento curricular e no acompanhamento dos estudantes em seu local de trabalho, na qualidade de professores colaboradores. Em contrapartida, docentes e estudantes participam do processo de trabalho das unidades de saúde, de forma a favorecer discussões e reflexões para a construção de um novo modelo de atenção.

A estrutura curricular de ambos os Cursos conta com as Unidades de Prática Profissional (UPP) e de Educacional Sistematizada (UES), que têm como propósito promover o desenvolvimento de recursos cognitivos, afetivos e psicomotores que possam ser mobilizados e integrados para a realização das tarefas propostas, de 
acordo com o domínio e autonomia dos estudantes em cada série.

Na UPP visa-se à identificação de necessidades de saúde de indivíduos, de famílias e de coletividades, por meio do confronto dos estudantes com a realidade dos serviços de saúde e, num movimento reflexivo, a busca de sua transformação. A UES segue a metodologia da Aprendizagem Baseada em Problemas (ABP) e busca oportunizar maior elaboração de processos cognitivos relacionados a situações-problema apresentadas em diferentes cenários de aprendizagem com olhar ampliado para as necessidades de saúde dos usuários dos serviços.

O desenvolvimento curricular da $1^{\text {a }}$ e da $2^{\text {a }}$ séries é compartilhado pelos Cursos de Enfermagem e Medicina, tanto na UPP como na UES, contribuindo para essa construção conjunta o referencial das necessidades em saúde das pessoas, da família e da coletividade, a fim de proporcionar uma visão ampliada de saúde, além de desenvolver a capacidade de trabalhar em equipe.

Em que pesem as potencialidades decorrentes da integração ensino-serviço nessa proposta de desenvolvimento curricular e a despeito do incentivo para a renovação dos processos de ensino e cuidado em saúde, observam-se na prática algumas dificuldades relacionadas à forma de inserção docente nos serviços, aos valores distintos atribuídos às atividades de ensino e de cuidado pelos diferentes sujeitos, além das dificuldades inerentes ao próprio processo de mudança, uma vez que princípios e práticas tradicionais encontram-se arraigados no imaginário dos envolvidos. A tais aspectos, acrescentam-se as mudanças da gestão municipal, pois, em cada mandato, são atribuídos valores e incentivos distintos à integração docente-assistencial.

Estudos que analisaram a integração ensinoserviço destacam que sua efetividade depende de mudanças estruturais e funcionais significativas, da construção conjunta entre gestores, trabalhadores, docentes e estudantes, com franca aproximação de sujeitos do mundo do trabalho e do ensino, dois universos que nem sempre dialogam suficientemente para que ocorra a troca e a complementariedade dos saberes ${ }^{10,11}$. Além disso, acredita-se na necessidade da apropriação de conhecimentos referentes à interdisciplinaridade, à transversalidade e à equidade entre os sujeitos envolvidos no processo ${ }^{12}$.

Outro aspecto considerado é a necessidade de fortalecimento de ações colaborativas tanto por parte do docente quanto dos profissionais assistenciais, devido à relevância de ambos no processo de ensino e aprendizagem e na transformação das práticas em saúde ${ }^{13}$. Destaca-se ainda o papel do professor colaborador na interlocução entre os diferentes interesses institucionais, no estímulo à participação ativa do estudante e no envolvimento da coletividade no planejamento das ações em saúde ${ }^{14}$.

Frente ao exposto, a questão que ora se coloca para a Famema é: Como o processo de integração ensino-serviço instituído tem favorecido mudanças na prática profissional e, por conseguinte, nas práticas de saúde na Atencão Básica, em consonância com o modelo de atenção proposto pelo SUS?

Observa-se na literatura escassez de trabalhos direcionados à temática, o que torna este estudo importante por propiciar o desenvolvimento de conhecimentos e sua difusão no que se refere à formação em saúde, visando dar continuidade às iniciativas governamentais expressas em políticas de saúde e educação, subsidiando a melhoria dos programas de formação profissional.

Dada a relevância da integração ensino-serviço na formação dos profissionais de saúde, o presente estudo propõe-se a identificar contribuições e limites da integração ensino-serviço da Famema, em parceria com SMSM, para a academia, para os serviços e para a coletividade.

\section{Método}

Trata-se de um estudo transversal, quantitativo, que analisa a integração ensino-serviço desenvolvida na Famema pela ótica dos sujeitos envolvidos (estudantes, docentes e professores colaboradores). A Famema é uma instituição pública estadual e oferece anualmente 40 vagas no Curso de Enfermagem e 80 no de Medicina.

A amostra do estudo foi composta pelos estudantes do $2^{\circ}$ ano do curso de Enfermagem e de Medicina que desenvolviam atividades na ESF, bem como por estudantes do $4^{\circ}$ ano de Enfermagem e do $5^{\circ}$ ano de Medicina, que já haviam vivenciado essas atividades, num total de 240 estudantes. Quanto aos docentes e professores colaboradores, foram incluídos todos aqueles que haviam desenvolvido atividades na UPP por um período mínimo de um ano, perfazendo 60 pessoas.

$\mathrm{O}$ instrumento de coleta de dados foi um questionário com 20 assertivas sobre a contribuição da integração ensino-serviço para a comunidade, para as equipes de saúde e para a formação profissional, em consonância com os princípios e diretrizes do SUS. Foi utilizada a escala 
Likert, com cinco possibilidades de respostas (1. discordo totalmente; 2 . discordo; 3 . indiferente; 4. concordo e 5. concordo totalmente).

O questionário foi respondido por 27 $(14,8 \%)$ docentes e professores colaboradores e $156(85,2 \%)$ estudantes, totalizando 183 participantes. Entre os estudantes, 90 (57,7\%) estavam cursando as e segundas séries dos Cursos e os demais a quarta série do Curso de Enfermagem ou a quinta do Curso de Medicina.

Para análise dos dados foram calculadas as proporções das variáveis ordinais e a moda. Além disso, foi realizada a comparação entre as respostas obtidas entre os diferentes participantes do estudo (estudantes, docentes e professores colaboradores) por meio do teste de Mann-Whitney.

A proposta de investigação foi submetida à aprovação do Comitê de Ética em Pesquisa com Seres Humanos da Famema. Os sujeitos foram esclarecidos quanto à finalidade do estudo e os que concordaram em participar assinaram o Termo de Consentimento Livre e Esclarecido.

\section{Resultados}

A Tabela 1 reúne as manifestações dos participantes às assertivas em três grandes grupos: contribuições para a formação profissional ( 1 a 7 ), para a comunidade ( 8 a 15 ) e para as equipes de saúde (16 a 20).

Verifica-se que as questões referentes às contribuições da integração ensino-serviço para a formação profissional foram as que apresentam maior tendência de avaliação positiva (concordo e concordo plenamente), destacando-se os itens 1 (Capacita o estudante para trabalhar em equipe) e 3 (Coloca o estudante em situações que fazem parte do papel do profissional da saúde), considerados positivos por $75 \%$ ou mais dos participantes da investigação. Por outro lado, a assertiva do item 6 (Desenvolve ações com outros setores da comunidade) foi a que obteve os menores percentuais de concordância.

Quanto às contribuições da integração ensino-serviço para a comunidade, embora no geral a avaliação positiva tenha se aproximado de $50 \%$, os itens 10 e 13 (Ajuda a resolver as necessidades de saúde dos usuários e Melhora a adesão à vacinação) foram os mais bem avaliados, enquanto nos itens 8 e 14 (Facilita o acesso dos usuários aos diferentes niveis de atenção a saúde e Melhora a participação do usuário nas atividades educativas em grupo), a avaliação foi mais negativa.
Os itens referentes às contribuições da integração para as equipes de saúde foram os que tiveram mais respostas negativas (discordo totalmente, discordo), especialmente o 17 (Favorece a educação permanente dos profissionais do serviço) e o 19 (Aumenta a satisfação dos trabalhadores do serviço). Nos demais, a avaliação positiva aproximou-se de $50 \%$.

As respostas de docentes e professores colaboradores foram comparadas às dos estudantes, utilizando-se o teste de Mann-Whitney. Foram constatadas divergências estatisticamente significativas ( $\mathrm{p} \leq \mathrm{a} 0,05)$ nos itens $3,4,10,11,12$, $14,15,17$ e 18 . As diferenças mais frequentes ocorreram nas assertivas relacionadas às Contribuições da IES para a comunidade. Em todos os aspectos em que a diferença foi estatisticamente significativa, as respostas de docentes e professores colaboradores foram mais favoráveis que as dos estudantes.

Na comparação entre estudantes de séries iniciais e finais foram encontradas diferenças estatisticamente significativas nos itens 1, 3, 4, 19 e 20 (Capacita o estudante para trabalhar em equipe, coloca o estudante em situações que fazem parte do papel do profissional da saúde, melhora a qualidade dos profissionais formados, aumenta a satisfação dos trabalhadores do serviço, contribui para a mudança das práticas profissionais e da organização do trabalho, respectivamente), com maior proporção de estudantes dos anos finais que concordaram ou concordaram plenamente com a assertiva dos itens $(87,3 \%$ versus $71,4 \%$, p $=0,001), 3(76,2 \%$ versus $68,6 \%, p=0,02)$ e 4 $(71,4 \%$ versus $53,9 \%, \mathrm{p}=0,03)$ e 20 (65\% versus $34,9 \% \%, p=0,01)$.

\section{Discussão}

Os dados revelam que a integração ensino-serviço contribui para a formação de profissionais em consonância com os princípios e diretrizes do SUS. Entretanto, expõem algumas fragilidades importantes a serem superadas.

A aproximação dos estudantes com os serviços da Atenção Básica, desde as primeiras séries dos Cursos, indicada como um aspecto positivo da integração, foi valorizada igualmente por estudantes, docentes e professores colaboradores.

$\mathrm{Na}$ ESF, o trabalho em territórios adstritos leva à possibilidade do estabelecimento de vínculo e responsabilização, uma vez que cada sujeito adentra à unidade contando com uma equipe 
Tabela 1. Contribuições da integração ensino-serviço para a formação profissional, para a comunidade e para as equipes de saúde, de acordo com o percentual de respostas ao questionário (escala de Likert). Marília, 2012.

\begin{tabular}{|c|c|c|c|c|c|}
\hline Questões & $\begin{array}{l}\text { Discordo } \\
\text { totalmente }\end{array}$ & Discordo & Indiferente & Concordo & $\begin{array}{l}\text { Concordo } \\
\text { totalmente }\end{array}$ \\
\hline \multicolumn{6}{|l|}{ Contribuições para a formação profissional } \\
\hline 1. Capacita o estudante para trabalhar em equipe & $3(1,6 \%)$ & $13(7,1 \%)$ & $20(10,9 \%)$ & $123(67,2 \%)$ & $24(13,1 \%)$ \\
\hline $\begin{array}{l}\text { 2. Sensibiliza o estudante para atuação como } \\
\text { futuro profissional em equipes da ESF }\end{array}$ & $11(6,0 \%)$ & $34(18,6 \%)$ & $30(16,4 \%)$ & $89(48,6 \%)$ & $18(9,8 \%)$ \\
\hline $\begin{array}{l}\text { 3. Coloca o estudante em situações que fazem } \\
\text { parte do papel do profissional da saúde. }\end{array}$ & $5(2,7 \%)$ & $18(9,8 \%)$ & $23(12,6 \%)$ & $103(56,3 \%)$ & $34(18,6 \%)$ \\
\hline 4. Melhora a qualidade dos profissionais formados. & $8(4,4 \%)$ & $14(7,7 \%)$ & $41(22,4 \%)$ & $102(55,7 \%)$ & $17(9,3 \%)$ \\
\hline $\begin{array}{l}\text { 5. Aproxima os profissionais de enfermagem e } \\
\text { medicina dos diferentes cenários da atenção à saúde. }\end{array}$ & $14(7,7 \%)$ & $30(16,4 \%)$ & $39(21,3 \%)$ & $82(44,8 \%)$ & $16(8,7 \%)$ \\
\hline $\begin{array}{l}\text { 6. Desenvolve ações com outros setores da } \\
\text { comunidade. }\end{array}$ & $13(7,1 \%)$ & $47(25,7 \%)$ & $49(26,8 \%)$ & $67(36,6 \%)$ & $5(2,7 \%)$ \\
\hline 7. Torna possível a interdisciplinaridade & $9(4,9 \%)$ & $21(11,5 \%)$ & $43(18,6 \%)$ & $105(57,4 \%)$ & $12(6,6 \%)$ \\
\hline \multicolumn{6}{|l|}{ Contribuições para a comunidade } \\
\hline $\begin{array}{l}\text { 8. Facilita o acesso dos usuários aos diferentes } \\
\text { níveis de atenção a saúde. }\end{array}$ & $13(7,1 \%)$ & $44(24,0 \%)$ & $51(27,9 \%)$ & $70(38,3 \%)$ & $5(2,7 \%)$ \\
\hline 9. Melhora o vínculo do usuário com o serviço. & $6(3,3 \%)$ & $24(13,1 \%)$ & $39(21,3 \%)$ & $107(58,5 \%)$ & $7(3,8 \%)$ \\
\hline $\begin{array}{l}\text { 10. Ajuda a resolver as necessidades de saúde dos } \\
\text { usuários. }\end{array}$ & $10(\%)$ & $16(8,7 \%)$ & $35(19,1 \%)$ & $111(60,7 \%)$ & $11(6,0 \%)$ \\
\hline $\begin{array}{l}\text { 11. Melhora a adesão do usuário ao tratamento } \\
\text { medicamentoso. }\end{array}$ & $7(3,3 \%)$ & $23(12,6 \%)$ & $59(32,2 \%)$ & $86(47,0 \%)$ & $7(3,8 \%)$ \\
\hline $\begin{array}{l}\text { 12. Melhora a adesão do usuário aos exames de } \\
\text { prevenção do câncer cérvico-uterino. }\end{array}$ & $5(2,7 \%)$ & $20(10,9 \%)$ & $62(33,9 \%)$ & $88(48,1 \%)$ & $8(4,4 \%)$ \\
\hline 13. Melhora a adesão do usuário à vacinação. & $7(3,8 \%)$ & $18(9,8 \%)$ & $35(19,1 \%)$ & $114(62,3 \%)$ & $9(4,9 \%)$ \\
\hline $\begin{array}{l}\text { 14. Melhora a participação do usuário nas } \\
\text { atividades educativas em grupo. }\end{array}$ & $9(4,9 \%)$ & $23(12,6 \%)$ & $67(36,6 \%)$ & $75(41,0 \%)$ & $7(3,8 \%)$ \\
\hline $\begin{array}{l}\text { 15. Promove hábitos de vida saudáveis } \\
\text { (alimentação adequada, atividades físicas). }\end{array}$ & $13(7,1 \%)$ & $24(13,1 \%)$ & $42(23,0 \%)$ & $92(50,3 \%)$ & $8(4,4 \%)$ \\
\hline \multicolumn{6}{|l|}{ Contribuições para as equipes de saúde } \\
\hline 16. Facilita o trabalho dos profissionais do serviço. & $8(4,4 \%)$ & $24(13,1 \%)$ & $49(26,8 \%)$ & $87(47,5 \%)$ & $12(6,6 \%)$ \\
\hline $\begin{array}{l}\text { 17. Favorece a educação permanente dos } \\
\text { profissionais do serviço. }\end{array}$ & $13(7,1 \%)$ & $38(20,7 \%)$ & $64(35,0 \%)$ & $56(30,6 \%)$ & $8(4,4 \%)$ \\
\hline $\begin{array}{l}\text { 18. Contribui para que a equipe de saúde conheça } \\
\text { melhor as necessidades de saúde das pessoas, das } \\
\text { famílias e da comunidade. }\end{array}$ & $6(3,3 \%)$ & $26(14,2 \%)$ & $29(15,8 \%)$ & $100(54,6 \%)$ & $19(10,4 \%)$ \\
\hline $\begin{array}{l}\text { 19. Aumenta a satisfação dos trabalhadores do } \\
\text { serviço. }\end{array}$ & $16(8,7 \%)$ & $52(28,4 \%)$ & $81(44,3 \%)$ & $29(15,8 \%)$ & $2(1,1 \%)$ \\
\hline $\begin{array}{l}\text { 20. Contribui para a mudança das práticas } \\
\text { profissionais e da organização do trabalho. }\end{array}$ & $8(4,4 \%)$ & $30(16,4 \%)$ & $52(28,4 \%)$ & $79(42,2 \%)$ & $10(5,5 \%)$ \\
\hline
\end{tabular}

que conhece seu contexto de vida e suas necessidades, o que facilita o processo de intervenção de forma integral e humanizada.

A tais resultados acrescentam-se dados que indicam que a integração ensino-serviço contribui para um olhar abrangente do processo saúde/doença, para o conhecimento do trabalho em equipe e seu funcionamento e, ainda, das neces- sidades dos serviços de saúde ${ }^{15,16}$; consolidação da relação teoria-prática, formação crítico-construtivista $^{17}$, oportunidade de desenvolver e aperfeiçoar habilidades de cuidado, educação, gerência e pesquisa ${ }^{18}$.

Salientam-se também avanços na compreensão da interdisciplinaridade e do funcionamento da rede de serviços de saúde ${ }^{19}$; reconhecimen- 
to do perfil epidemiológico local, identificação dos problemas e direcionamento das intervenções ${ }^{20}$. Todos esses aspectos favorecem a aprendizagem significativa e o desenvolvimento de pesquisas a partir da realidade, servindo para uma formação profissional mais humanizada e contextualizada com a prática profissional ${ }^{14,20}$.

Em um estudo sobre a integração ensino-serviço entre um curso de fisioterapia e um serviço da Atenção Básica, a formação, antes voltada para a reabilitação, passou a focar a prevenção do adoecimento e a promoção da saúde ${ }^{15}$. Em um curso de medicina, houve grande aproximação com a comunidade e com a forma de organização da Atenção Básica ${ }^{16}$.

É necessário reconhecer, no entanto, que tais avanços, por si só, não garantem que o ensino seja integralmente pautado em uma nova lógica de atenção, uma vez que nos serviços de saúde ainda prevalece o atendimento à demanda e à queixa principal. Assim, o percurso em prol de novas formas de agir e de pensar na formação em saúde e no cuidado situa-se entre as propostas de inovação do modelo de atenção tradicional ainda presente no cotidiano dos serviços.

A atual proposta de atenção à saúde do SUS difere radicalmente do modelo que prevaleceu na realidade de saúde no Brasil, por muitas décadas, sem conseguir responder adequadamente às necessidades de saúde da população. Esse modelo hegemônico tem como características o atendimento individual, o fato de ser centrado na queixa e nos aspectos biológicos, a fragmentação do cuidado e o hospital como principal cenário de atenção. Portanto, não se pode esperar que as transformações ocorram de imediato e de forma completa, pois envolvem mudanças de paradigma e requerem uma compreensão do processo saúde-doença que se diferencia do que se encontra no imaginário das pessoas, sejam elas estudantes, docentes, profissionais, gestores ou usuários dos serviços de saúde.

Neste sentido, as respostas obtidas no item seis (Desenvolve ações com outros setores da comunidade), em grande proporção, aproximamse de uma avaliação negativa (discordo totalmente e discordo).

Vale ressaltar que a formação com foco na Atenção Básica deve privilegiar o refinamento comunicativo e relacional ${ }^{14}$, uma vez que atuar nesse cenário requer habilidades de negociação e pactuação, pois se trata de um contexto de grande complexidade e diversidade, que exige uma prática reflexiva dos profissionais para lidar com situações não capturadas pela lógica biomédica ${ }^{14}$.
No presente estudo, algumas assertivas que traduziam as contribuições da integração ensino-serviço para a comunidade tiveram avaliação com tendência positiva como, por exemplo, a melhoria do vínculo do usuário com o serviço, a ajuda na resolução das necessidades de saúde e a melhoria na adesão à vacinação.

Como condição que possibilita a complementação das atividades desenvolvidas junto à comunidade, a integração ensino-serviço também representa um avanço na direção de transformações articuladas entre o ensino e os serviços, o que por certo se reflete na qualidade da atenção à saúde.

Corrobora tais achados a explicitação de que a integração ensino-serviço possibilita melhoria da qualidade do cuidado, pois contribui para um olhar abrangente do paciente e do processo de adoecer, além de contribuir para a melhoria das condições de vida ${ }^{19,21}$. Um programa de atenção à criança, desenvolvido na Atenção Básica pelo Programa de Integração Docente Assistencial, resultou em melhoria das condições de vida das crianças atendidas ${ }^{22}$.

Por outro lado, foram avaliadas, com tendência negativa, a facilitação do acesso e a melhoria da participação dos usuários nas atividades em grupo, o que nos leva a refletir sobre as mudanças organizacionais e estruturais necessárias ${ }^{23}$ nos processo de trabalho para que haja coerência entre o projeto dos serviços e a proposta educativa, nas dimensões política, técnica e metodológica, com o envolvimento dos gestores dos diferentes cenários e da população ${ }^{24}$.

No que se refere às contribuições da integração ensino-serviço para as equipes de saúde, entre os aspectos analisados, destacaram-se as assertivas favorece a educação permanente dos profissionais do serviço; aumenta a satisfação dos trabalhadores dos serviços e contribui para a mudança das práticas profissionais e da organização do trabalho, cuja avaliação foi predominantemente negativa ou indiferente.

Dessas respostas pode-se depreender que persistem desafios para a plena implantação da integração ensino-serviço, uma vez que a falta de reflexão crítica sobre o processo de trabalho instituído leva à manutenção do cuidado em seu propósito produtivo, desenvolvido por meio de modalidades técnicas. Também vale questionar se a forma de inserção dos estudantes nos serviços vem ao encontro da prática dos profissionais que, muitas vezes, se encontram envolvidos em uma dinâmica complexa. É preciso levar em conta que os serviços de Atenção Básica são a 
porta de entrada ao sistema de saúde, muitas vezes com pouco respaldo dos demais níveis de atenção à saúde para a resolução das necessidades dos usuários.

Referindo-se à formação médica na perspectiva da integralidade, alguns dos participantes ressaltaram a necessidade de ressignificar o papel da academia, com vistas a uma interlocução mais horizontalizada com os serviços de saúde. Destacaram ainda que a inserção dos estudantes nos serviços deve favorecer a problematização do processo de trabalho, visando ao aperfeiçoamento do trabalho coletivo e à superação dos obstáculos à qualificação das ações no SUS ${ }^{14}$.

Para as equipes, a integração deve possibilitar a capacitação dos profissionais por meio da educação permanente e da troca de informações, preferencialmente no processo de trabalho cotidiano. Assim, revela-se que a inserção dos docentes em atividades assistenciais tem permitido o intercâmbio de experiências com os profissionais, que também puderam participar de cursos de aperfeiçoamento ou atualização ${ }^{25}$ e de curso de pós-graduação senso estrito ${ }^{26}$. A presença do estudante no cenário de prática também foi considerada favorável para o aprimoramento crítico e reflexivo dos profissionais do serviço ${ }^{10}$. No caso específico da Famema, não se pode afirmar que este aspecto tenha se efetivado a contento, visto que nas respostas dos participantes do estudo, no que se refere à educação permanente, tenderam à indiferença e à avaliação negativa.

Ainda, no que se refere às contribuições da integração ensino-serviço para a equipe, é preciso considerar a avaliação negativa e indiferente atribuída pelos integrantes ao item aumenta a satisfação dos trabalhadores do serviço, o que pode estar relacionado com a pequena possibilidade de trocas efetivas, embora tal relação careça de estudo mais aprofundado.

Outro aspecto que demanda maior compreensão e aprofundamento é a avaliação mais positiva efetuada por docentes, professores cola- boradores e estudantes de séries finais, em comparação com a dos estudantes das séries iniciais.

\section{Considerações finais}

Na análise da integração ensino-serviço, a avaliação mais positiva refere-se às contribuições para a formação profissional. Ao possibilitar a inserção precoce do estudante no contexto do trabalho da Atenção Básica e o processo de ensino e aprendizagem a partir da prática profissional vivenciada no cotidiano desses serviços, a Famema e a SMSM contemplam as diretrizes de formação dos profissionais da saúde e os princípios e diretrizes do SUS.

No entanto, não se pode afirmar que tal avanço tenha sido suficiente para que o estudante vivencie uma nova modalidade de atenção à saúde, uma vez que, embora a ESF apresente características importantes nesta construção, a lógica da atenção ainda se encontra voltada para o atendimento da demanda. Outro aspecto a ser considerado é a contribuição inexpressiva dessa integração para a educação permanente e para a satisfação dos trabalhadores da equipe.

Assim, decorrida mais de uma década da sua implantação, a integração ensino-serviço envolvendo a Famema e a SMSM ainda enfrenta desafios importantes a serem superados. Em contrapartida, não se pode negar que se trata de uma iniciativa importante para a inovação no campo da formação de médicos e enfermeiros e a transformação das práticas em saúde.

Os resultados deste estudo indicam que é preciso avançar de forma que ensino e serviço sejam considerados como processos interdependentes e com possibilidades de reflexões conjuntas, visando às intervenções efetivas em prol de uma nova lógica de atenção à saúde. Além disso, sugerem a necessidade de novos estudos com vistas a aprofundar a compreensão da integração ensino-serviço no contexto da Atenção Básica.

\section{Colaboradores}

MJS Marin coordenou o projeto e participou de todas as etapas do estudo. MAC Oliveira, MAP Otani, CP Cardoso, MYAD Moravcik, LO Conterno, LAD Braccialli, CRR Nunes e AC Siqueira Júnior participaram igualmente de todas as etapas de elaboração da pesquisa. 


\section{Referências}

1. Pinto LLS, Formigli VLA, Rêgo RCF. A dor e a delícia de aprender com o SUS: integração ensinoserviço na percepção dos internos em medicina social. Rev Baiana Saúde Pública 2007; 3(1):115-133.

2. Campos GWS. Educação médica, hospitais universitários e o Sistema Único de Saúde. Cad Saude Publica 1999; 15(1):187-193.

3. Brasil. Constituição da República Federativa do Brasil de 1988. Diário Oficial da União 1988; 5 out.

4. Brasil. Ministério da Saúde (MS), Organização Mundial da Saúde, Organização Pan-Americana da Saúde. Políticas de recursos humanos em saúde: seminário internacional. Brasília: MS; 2002.

5. Faustino RLH, Moraes MJB, Oliveira MAC, Egry EY. Caminhos da formação de enfermagem: continuidade ou ruptura? Rev Bras Enferm 2003; 56(4): 343-347.

6. Ceccim RB, Feuerwerker LCM. Mudança na graduação das profissões de saúde sob o eixo da integralidade. Cad Saude Publica 2004; 20(5):1400-1410.

7. Bourget MMM, organizadora. Programa Saúde da Família: guia para o planejamento local. São Paulo: Martinari; 2005.

8. Sacristán JG. O currículo: uma reflexão sobre a prática. 3a Edição. Porto Alegre: Artmed; 2000.

9. Faculdade de Medicina de Marília. Necessidades de saúde: $1^{\text {a }}$ e $2^{\text {a }}$ série: cursos de medicina e enfermagem. Marília: Faculdade de Medicina de Marília; 2007.

10. Spagnol CA, Silva CMMF, Figueiredo ES, Nascimento JCM, Santos MBA, Donoso MTV, Canhestro MR, Santos ARG. Comissão de estágios em enfermagem: a experiência do Hospital das Clínicas da Universidade Federal de Minas Gerais. REME Rev Min Enferm 2004; 8(2):326-329.

11. Morais FRR, Leite IDR, Oliveira LL, Verás RM. A reorientação do ensino e da prática de enfermagem: implantação do Pró-Saúde em Mossoró, Brasil. Rev Gauch Enferm 2010; 31(3):442-449.

12. Lobo MSC, Lins MPE, Silva ACM, Fiszman R. Avaliação de desempenho e integração docente-assistencial nos hospitais universitários. Rev Saude Publica 2010; 44(4):581-590.

13. Dias HS. A implementação da política de reorientação da formação em odontologia: dependência de trajetória e estímulos institucionais na UFBA [dissertação]. Rio de Janeiro: Escola Nacional de Saúde Pública Sérgio Arouca; 2011.

14. Mestriner Júnior W, Mestriner SF, Bulgarelli AF, Mishima SM. O desenvolvimento de competências em atenção básica à saúde: a experiência no projeto Huka-Katu. Cien Saude Colet 2011; 16(Supl. 1):903912.
15. Ribeiro KSQS. A contribuição da extensão comunitária para a formação acadêmica em fisioterapia. Fisioter Pesqui 2005; 12(3):22-29.

16. Oliveira MS, Duarte JES, Baaklini CE, Padilha RQ. O PSF como cenário de ensino-aprendizagem em Marília (SP). Divulg Saúde Debate 2004; (30):99-102.

17. Sisson MC. Implantação de programas e redefinição de práticas profissionais. Rev Bras Educ Med 2009; 33(Supl. 1):92-103.

18. Campos MAF, Forster AC. Percepção e avaliação dos alunos do curso de medicina de uma escola médica pública sobre a importância do estágio em saúde da família na sua formação. Rev Bras Educ Med 2008; 32(1):83-89.

19. Bonin JE, Oliveira JGS, Nascimento JM, Rezende ME, Stopato SP, Leite ICG. Liga acadêmica de medicina de família e comunidade: instrumento de complementação curricular. Rev APS 2011; 14(1):50-57.

20. Lima EP. Epidemiologia e estatística: integrando ensino, pesquisa, serviço e comunidade. Rev Bras Educ Med 2010; 34(2):324-328.

21. Puccini RF. A integralidade na atenção à saúde da criança e o ensino de pediatria [livre docente]. São Paulo: Universidade Federal de São Paulo; 2002.

22. Nuto SAS, Oliveira GC, Andrade JV, Maia MCG. O Acolhimento em saúde bucal na estratégia de saúde da família, Fortaleza/CE: um relato de experiência. Rev APS 2010; 13(4):505-509.

23. Santos WS. Organização curricular baseada em competência na educação médica. Rev Bras Educ Med 2011; 35(1):86-92.

24. Pereira JG, Fracolli LA. Articulação ensino-serviço e vigilância da saúde: a percepção de trabalhadores de saúde de um distrito escola. Trab Educ Saúde 2011; 9(1):63-75.

25. Caetano JA, Diniz RCM, Soares E. Integração docente-assistencial sob a ótica dos profissionais de saúde. Cogitare Enferm 2009; 14(4):638-644.

26. Beccaria LM, Trevizan MA, Janucci MZ. Integração docente-assistencial entre um curso de enfermagem e um hospital de ensino: concepção do processo sob a ótica de docentes, alunos e enfermeiros. Arq Ciênc Saúde 2006; 13(3):137-145.

Artigo apresentado em 24/06/2012

Aprovado em 26/10/2012

Versão final apresentada em 14/11/2012 